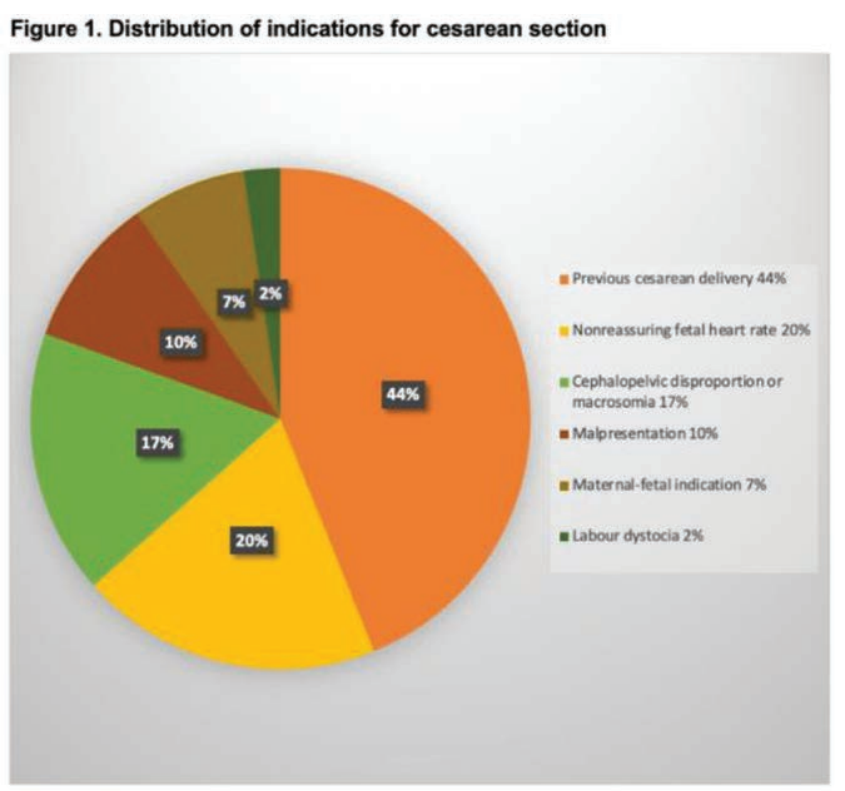

Disclosure of Interests: None declared

DOI: 10.1136/annrheumdis-2021-eular.3718

\section{AB0844 HAND GRIP STRENGTH EVALUATION IN RHEUMATOLOGIC DISEASES}

A. Caturano ${ }^{1}$, P. C. Pafundi ${ }^{1}$, R. Galiero ${ }^{1}$, M. Tardugno ${ }^{2}$, F. C. Sasso ${ }^{1}$, G. Cuomo ${ }^{2}$. 'University of Campania "Luigi Vanvitelli", Department of Advanced Medical and Surgical Sciences, Naples, Italy; ${ }^{2}$ University of Campania "Luigi Vanvitelli,' Department of Precision Medicine, Naples, Italy

Background: Handgrip muscle strength test describes the strength of the hand muscles used to grasp or grip. Currently, hand grip evaluation is often used in clinical practice, as a marker of function and disability. In fact, it has already been applied as an outcome measure in arthritis rheumatoid clinical trials, to demonstrate the benefits of several treatments [1]. However, hand disability should also be considered in all other rheumatological diseases.

Objectives: The main aim of this study is to assess the handgrip muscle strength test in a rheumatological cohort of patients as compared to a control group. Methods: This is a cross-sectional pilot study. We considered eligible 35 rheumatological consecutive female patients followed at our outpatients' clinic of Internal Medicine (I Policlinico of Naples) and 35 healthy control females (HC). Both groups included only right-handed individuals. Rheumatological patients were distributed as follows: 5 rheumatoid arthritis $(14,3 \%), 9$ psoriatic arthritis $(25,7 \%)$, 4 systemic lupus erythematosus $(11,4 \%), 10$ systemic sclerosis $(28,6 \%), 4$ fibromyalgia $(11,4 \%)$, 3 juvenile idiopathic arthritis $(8,6 \%)$. The course of disease was under optimal treatment in all patients.

The type of hand grip used is the power grip, in which an object is held firmly by wrapping the fingers around it, pressing the object against the palm, and using the thumb to apply counter-pressure. We considered as either right or left hand valid measure the mean of three consecutive tests per arm. Between-groups differences were tested both by a uni- and multivariable analysis.

Results: The two subgroups were homogeneously distributed for age (median age 42 yrs. [IQR 33-48] vs. 36 yrs. [IQR 30-52] in $\mathrm{HC} ; \mathrm{p}=0.902$ ). At univariate analysis, hand grip strength was significantly lower in the rheumatological patients, both at right hand (right $19.5 \mathrm{~kg}$ [IQR 13.6-24.8] vs. $24.5 \mathrm{~kg}$ [IQR 20.8-29] in HC; $\mathrm{p}=0.004$ ) and left hand (18.5 kg [IQR 13.9-22.5] vs. $23.7 \mathrm{~kg}$ [IQR 19-27.3] in HC; $\mathrm{p}=0.002$ ), as compared to $\mathrm{HC}$. This finding was further confirmed at multivariable analysis only as for the left hand (OR $0.919,95 \% \mathrm{Cl}: 0.858-0.984 ; \mathrm{p}=0.016$ ).

Conclusion: Rheumatological diseases are burdened by hand disability, mostly affecting daily activities performance [2,3]. Beyond an optimal disease control, our pilot study shows a decrease in left hand strength as compared to healthy controls. This might be due to a reduced use of the non-dominant hand, which may lead over time to a higher deficit of strength. As such, these patients should be prescribed to a left hand exercise to improve both mobility and strength and, consequently, hand function.

REFERENCES:

[1] Eberhardt K, Sandqvist G, Geborek P (2008) Hand function tests are important and sensitive tools for assessment of treatment response in patients with rheumatoid arthritis. Scand J Rheumatol 37(2):109-112.
[2] Feced Olmos CM, Alvarez-Calderon O, Hervás Marín D, et al. Relationship between structural damage with loss of strength and functional disability in psoriatic arthritis patients. Clin Biomech (Bristol, Avon). 2019 Aug;68:169174. doi: 10.1016/j.clinbiomech.2019.06.009.

[3] Maddali-Bongi S, Del Rosso A, Mikhaylova S, et al. Impact of hand and face disabilities on global disability and quality of life in systemic sclerosis patients. Clin Exp Rheumatol. 2014 Nov-Dec;32(6 Suppl 86):S-15-20.

Disclosure of Interests: None declared

DOI: 10.1136/annrheumdis-2021-eular.3860

\section{AB0845 THE COMBINATION OF PLASMA FIBRINOGEN CONCENTRATION AND NEUTROPHIL-LYMPHOCYTE RATIO (F-NLR) AS A NOVEL INFLAMMATORY MARKER OF RHEUMATOID ARTHRITIS}

M. Liu ${ }^{1}$, Z. Huang ${ }^{1}$, Y. Huang ${ }^{1}$, Z. Huang ${ }^{1}$, Q. Huang ${ }^{1}$, T. W. LI ${ }^{1} .{ }^{1}$ Guangdong Second Provincial General Hospital, Department of Rheumatology and Immunology, Guangzhou, China

Background: The combined index of fibrinogen and neutrophil-lymphocyte ratio (F-NLR) has recently been reported as a new predictive factor in patients with cancer. However, the fibrinogen and NLR have not been simultaneously evaluated in rheumatoid arthritis (RA).

Objectives: This study aimed to explore the clinical value of F-NLR in RA and its relationship with disease activity.

Methods: This retrospective study collected 143 RA patients and 82 age- and gender-matched healthy controls. Neutrophil, lymphocyte, monocyte, platelet, fibrinogen, NLR, monocyte to lymphocyte ratio (MLR), platelet to lymphocyte ratio (PLR), C-reactive protein (CRP), erythrocyte sedimentation rate (ESR), Disease Activity Score of 28 joints-ESR (DAS28-ESR) and other laboratory parameters were recorded. Receiver operating characteristic $(\mathrm{ROC})$ curves were used to defined the optimization cut-off values of fibrinogen and NLR, which were $3.9 \mathrm{~g} / \mathrm{L}$ and 2.42. The F-NLR score was 2 for patients with high fibrinogen $(>3.9 \mathrm{~g} / \mathrm{L})$ and elevated NLR (>2.42), while those with one or neither were indexed as 1 or 0 . The correlations between F-NLR as well as other inflammatory indexes and DAS28-ESR were measured.

Results: The F-NLR score was higher in RA patients than that in healthy individuals $(P<0.05)$. The proportion of higher F-NLR score increased significantly along with the disease activity $(P<0.05)$. According to the ROC curve which was conducted to discriminate RA patients from healthy subjects, the area under curve (AUC) of F-NLR $(0.803,95 \% \mathrm{Cl}: 0.744-0.861)$ was higher than that of fibrinogen (0.735, 95\% Cl: 0.670 - 0.801), NLR $(0.724,95 \% \mathrm{Cl}: 0.655-0.794)$ $\operatorname{MLR}(0.687,95 \% \mathrm{Cl}: 0.615-0.759)$ and PLR $(0.732,95 \% \mathrm{Cl}: 0.664-0.800)$. Furthermore, F-NLR was more strongly associated with DAS28-ESR $(r=0.572, P<$ $0.001)$ when compared with fibrinogen $(r=0.518, P<0.001)$, NLR $(r=0.365, P<$ $0.001)$, MLR $(r=0.140, P=0.096), \operatorname{PLR}(r=0.239, P=0.004)$, CRP $(r=0.539$, $P<0.001)$ and ESR $(r=0.487, P<0.001)$.

Conclusion: The results demonstrated that the F-NLR score was elevated in RA patients. The F-NLR score may be a potential marker to monitor the disease activity of RA patients.

\section{REFERENCES:}

[1] Wang H, Zhao J, Zhang M, Han L, Wang M, Xingde L. The combination of plasma fibrinogen and neutrophil lymphocyte ratio (F-NLR) is a predictive factor in patients with resectable non small cell lung cancer. J Cell Physiol. 2018 May; 233(5):4216-4224.

Disclosure of Interests: None declared

DOI: 10.1136/annrheumdis-2021-eular.3929

\section{Education}

\section{AB0846}

CONCEPTION AND FEASIBILITY OF A DIGITAL TELEGUIDED ABDOMEN, THORAX AND THYROID GLAND ULTRASOUND COURSE FOR MEDICAL STUDENTS

E. Höhne ${ }^{1}$, F. Recker ${ }^{2}$, E. Schmok ${ }^{3}$, P. Brossart ${ }^{1}$, T. Raupach ${ }^{4}$, V. Schäfer ${ }^{1}$. ${ }^{1}$ University Hospital Bonn, Clinic of Internal Medicine III, Oncology, Hematology, Rheumatology and Clinical Immunology, Bonn, Germany; ${ }^{2}$ University Hospital Bonn, Department for Obstetrics and Gynecology, Bonn, Germany; ${ }^{3}$ Amboss GmbH, Institutional Partnerships, Berlin, Germany; ${ }^{4}$ University Hospital Bonn, Institute for Medical Education, Bonn, Germany

Background: Over the past few decades, technological advances in both ultrasound (US) and the application of telemedicine have been made [1]. Medical education has shifted to online classes during the COVID-19 pandemic, creating challenges in adequate training of US. 